\title{
Circulatory microRNA-145 expression is increased in cerebral ischemia
}

\author{
C.S. Gan ${ }^{1}$, C.W. Wang ${ }^{1}$ and K.S. Tan ${ }^{2}$ \\ ${ }^{1}$ Department of Molecular Medicine, Faculty of Medicine, \\ University of Malaya, Kuala Lumpur, Malaysia \\ ${ }^{2}$ Department of Medicine, Faculty of Medicine, University of Malaya, \\ Kuala Lumpur, Malaysia \\ Corresponding author: C.S. Gan \\ E-mail: mr.dennisgan@gmail.com
}

Genet. Mol. Res. 11 (1): 147-152 (2012)

Received July 21, 2011

Accepted October 10, 2011

Published January 27, 2012

DOI http://dx.doi.org/10.4238/2012.January.27.1

ABSTRACT. Cerebral ischemia or ischemic stroke is mainly attributed to vascular and circulation disorders. Among protein biomarkers, RNA profiles have also been identified as markers of ischemic stroke. MicroRNA-145 expression is ostensibly recognized as marker and modulator of vascular smooth muscle cell phenotype; however, expression levels in ischemic stroke had not been investigated. Employing real-time quantitative PCR, we examined the expression profile of circulatory microRNA-145 in healthy control subjects $(\mathrm{N}=$ $14)$ and ischemic stroke patients $(\mathrm{N}=32)$. Circulatory microRNA-145 expression was significantly higher in ischemic stroke patients than in control subjects. This demonstrates that hemostatic mechanisms are affected by ischemic stroke. We conclude that circulating microRNA-145 has potential as a biomarker for ischemic stroke.

Key words: Biomarker; Circulation; Gene expression; Ischemic stroke; MicroRNA-145; Postnatal neovascularization 


\section{INTRODUCTION}

Stroke is a leading cause of mortality and permanent disability, with two-thirds of onsets due to ischemia (Lloyd-Jones et al., 2009). A non-intrusive ancillary test to support conservative clinical diagnosis, classification and prognosis, aimed at improving stroke management is desirable. Protein biomarkers have been suggested and shown to be associated with the onset of cerebral ischemia, manifesting its potential use in clinical practice. Among many, C-reactive protein, interleukin-6, matrix metallopeptidase 9, vascular cell adhesion molecule 1 , and intercellular adhesion molecule 1 have been shown to be dysregulated in stroke studies (Jickling and Sharp, 2011). RNA-based studies also unveil promising biomarker candidates for stroke. However, research on these biomarkers is ongoing and they are not yet used in clinical settings.

MicroRNAs are a class of small, non-coding RNAs that have the affinity to pair with sites in 3' untranslated regions in mRNAs, orchestrating their expression (Lagos-Quintana et al., 2001). This posttranscriptional regulation of nucleotide expression by microRNAs makes these small RNA pieces intriguing candidate biomarkers for potential detection before phenotypic projection. It is noteworthy that specific microRNA expressions have been shown in both brain tissue and blood following ischemic stroke mimic (Jeyaseelan et al., 2008). Moreover, circulatory microRNAs manifest the potential to be developed as ischemic stroke biomarkers in diagnosis and prognosis (Tan et al., 2009).

MicroRNA-145 has been extensively studied and its role in modulating the oscillating state of smooth muscle cells has been elucidated (Cheng et al., 2009; Cordes et al., 2009). Vascular smooth muscle cells (VSMC) are a critical cellular constituent of the vascular structure. They exhibit remarkable plasticity and readily change phenotype in response to a plethora of extrinsic stimuli, including mechanical injury, growth factors and oxidative stress (Owens, 1995). Compiling evidence strongly associates intriguing microRNA-145 expression as a modulator of smooth muscle cell (SMC) phenotype. These studies revealed the inverse correlation of microRNA-145 level to the Kruppel-like factor (KLF) family of transcription factor protein transcripts, thus identifying microRNA-145 as a novel SMC phenotype biomarker (Cheng et al., 2009; Cordes et al., 2009). However, the expression profile of circulatory microRNA-145 in vascular and circulatory disorders remains to be explored. Thus, this study aimed to examine the expression profile of circulatory microRNA-145 of ischemic stroke patients. This profile will serve as a reference and contribution to the knowledge pertaining to circulatory microRNA-145 in ischemic stroke.

\section{MATERIAL AND METHODS}

\section{Sample collection and ethical statement}

Ischemic stroke patients $(\mathrm{N}=32)$ between the ages of 18 and 49 years were recruited among those admitted for neurology treatment at University of Malaya Medical Centre (UMMC). Ischemic stroke was confirmed by either MRI or CT imaging of the brain, and the risk factors, if any, were characterized based on the ancillary blood and routine tests (Tan, et al., 2009). Excluded from the study were subjects with hemorrhage stroke. The patients $(\mathrm{N}=11)$ were recruited for second sampling, and healthy volunteers $(\mathrm{N}=14)$ without risk factors or his- 
tory of cardiovascular and cerebrovascular diseases were included as control subjects. The study protocol was approved by the Medical Ethics Committee of UMMC (reference number 607.20). Each volunteer prior to blood sampling gave written informed consent.

\section{Total RNA isolation and preparation}

Total RNA was isolated from peripheral whole blood using the Ribopure-Blood RNA isolation kit (Ambion, Austin, TX, USA), adhering strictly to the recommended protocol by the manufacturers. The eluted RNA was quantified by NanoDrop ND-1000 Spectrophotometer (Rockland, DE, USA) and assessed for integrity by $1 \%$ denaturing agarose and $15 \%$ denaturing polyacrylamide gel electrophoresis.

\section{Quantification of microRNA-145}

Quantification of microRNA was carried out with the TaqMan Real-Time PCR. TaqMan MicroRNA Reverse Transcription kit and High Capacity RNA-to-cDNA Master Mix were used for reverse transcription of the RNA. TaqMan MicroRNA Assay (P/N 4373133) and Universal PCR Master Mix were used for real-time quantitative PCR. Ribosomal 18s rRNA was used as an endogenous control. RNA template (10 ng) was subjected to reverse transcription. The cDNA product $(1.33 \mathrm{~mL})$ was used for PCR. Real-time PCR was carried out using the StepOnePlus Real-Time PCR system (Applied Biosystems, Foster City, CA, USA). All procedures adhered to the protocols were provided by the manufacturer.

\section{Statistical analysis}

The Student $t$-test was performed using the SPSS software for Windows version 11.0, and the level of significance was set at $\mathrm{P}<0.05$. The data are reported as means \pm standard error of the mean.

\section{RESULTS AND DISCUSSION}

The circulatory microRNA-145 of patients, compared to that of controls, demonstrated significant upregulation $(\mathrm{P}=0.022$; Figure $1 \mathrm{~A})$. The altered expression profile of circulatory microRNA-145 in this study may mark the biological stages of vascular reendothelialization, a postnatal vasculogenesis response after cerebral ischemia (Dimmeler and Zeiher, 2004). The upregulation of circulatory microRNA-145 detected may derive from the pool of circulating progenitor cells that are in commitment to differentiate into VSMC lineage, since it has been shown that the introduction of microRNA-145 into neural crest stem cells is sufficient to propitiously guide the progenitor cells to differentiate into VSMC (Cordes et al., 2009). Thus, upregulation of circulatory microRNA-145 suggests positive reendothelialization, while downregulation or vice versa.

As has been delineated, microRNA-145 shows an inverse correlation with the expression of KLF4/5 proteins, regulating the mRNA transcripts, posttranscriptionally. High expression level of microRNA-145 will consequently down modulate its targets, KLF4/5; myocardin will not be suppressed and will form a complex with serum response factor, which eventually 
promotes the transcription of the genes essential for VSMC differentiation and maintenance of the differentiated state (Figure 2) (Long et al., 2008; Cheng et al., 2009; Cordes et al., 2009).
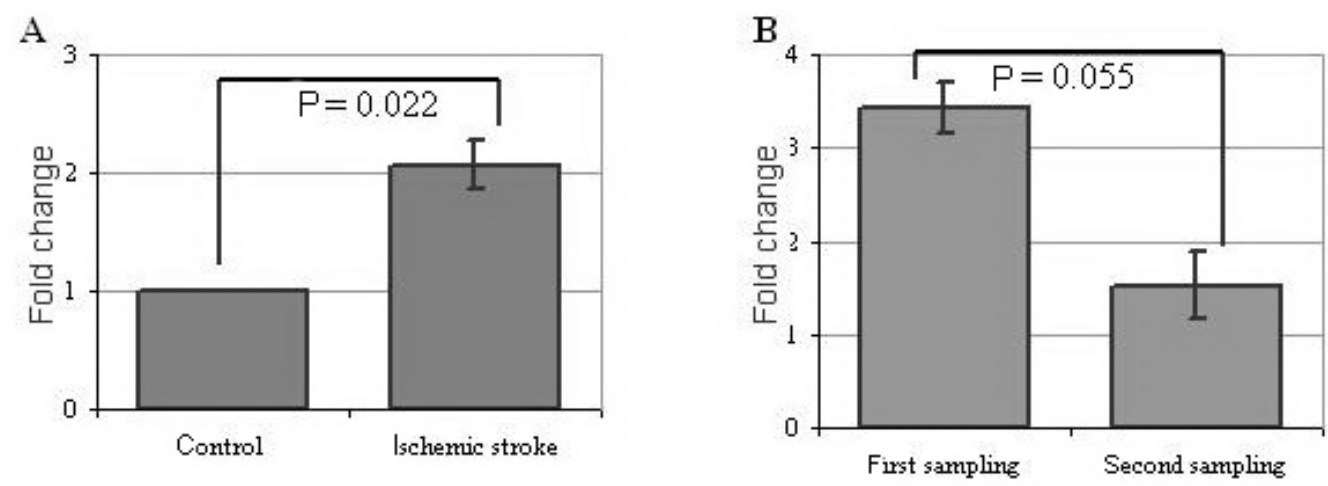

Figure 1. A. Circulatory microRNA-145 expression profile of ischemic stroke patients, $N=32$. B. Paired expression profile of patients, $\mathrm{N}=11$. Peripheral blood was resampled and expression profile of circulatory microRNA-145 was generated and compared. Expression values are normalized to the mean of the expression values from healthy controls. All values are reported as mean fold changes \pm standard error of the mean, and $\mathrm{P}<0.05$ is considered to be significant.

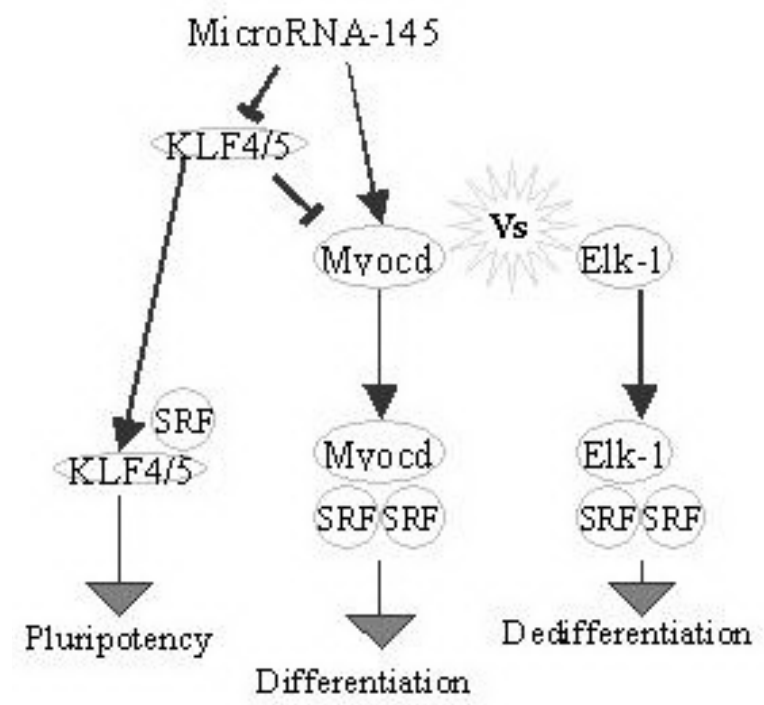

Figure 2. Effect of microRNA-145 congregate on serum response factor (SRF)-dependent transcription by regulation of co-activators and co-repressors directing cell fate. MicroRNA-145 potentiates myocardin (Myocd) and negatively regulates Kruppel-like factor (KLF4/5), which interacts with SRF and also inhibits Myocd. KLF is a transcription factor implicated in pluripotency. ETS-like transcription factor 1 (Elk-1) represses Myocd's activity by competing for common docking site (displacing Myocd from SRF), activating gene transcription for cell dedifferentiation. Upregulation of microRNA-145 renders Myocd functional in directing cell differentiation by repressing KLF4/5 and potentiates Myocd's effect over Elk-1 (Long et al., 2008; Cheng et al., 2009; Cordes et al., 2009). 
On the other hand, postnatal neovascularization is shown to be no longer attributed to angiogenesis only. Postnatal vasculogenesis has been described as also being involved in vascular homeostasis. This process is involved in the recruitment and incorporation of precursor cells, which have also been shown to circulate postnatally in the peripheral blood to the injured and ischemic sites (Luttun et al., 2002). Besides neural crest stem cells, bone marrow-derived circulating endothelial precursor cells, common vascular progenitors, and SMC progenitors have been shown to possess the potential to participate in postnatal vasculogenesis, extending the pool of circulating multipotent precursor cells in vascular regeneration (Luttun et al., 2002; Dimmeler and Zeiher, 2004). These findings support the observation in this study.

Therefore, plummet of the upregulated circulatory microRNA-145 may suggest a good outcome in the completion of vascular regeneration, achieving homeostatic equilibrium (Tan et al., 2009). Ischemic stroke patients $(\mathrm{N}=11)$ were called back months after the initial blood sampling. The pair expression profile of circulatory microRNA-145 (first sampling $v s$ second sampling) demonstrated nonsignificant downregulation $(\mathrm{P}=0.055$; Figure $1 \mathrm{~B})$. This may be due to the limitations of the study in that only some of the patients agreed to subsequent samplings or the time of second collection was too parsimonious for effective postnatal vasculogenesis, which is affected by individual risk factors (Dimmeler and Zeiher, 2004).

\section{CONCLUSION}

The present study revealed that circulatory microRNA-145 expression is upregulated in ischemic stroke patients, as compared to the control. This finding may have implications for the development of a desirable biomarker and therapy for ischemic stroke. Since this study utilized peripheral whole blood, identification of the source of the upregulated circulatory microRNA-145 would be beneficial for the elucidation of mechanotransduction.

\section{ACKNOWLEDGMENTS}

Research supported by the University Malaya Research Grant(UMRG, \#FS192/2008B) and the University Malaya Postgraduate Research Fund (PPP, \#PS385/2009B) for K.S. Tan and C.S. Gan, respectively.

\section{REFERENCES}

Cheng Y, Liu X, Yang J, Lin Y, et al. (2009). MicroRNA-145, a novel smooth muscle cell phenotypic marker and modulator, controls vascular neointimal lesion formation. Circ. Res. 105: 158-166.

Cordes KR, Sheehy NT, White MP, Berry EC, et al. (2009). miR-145 and miR-143 regulate smooth muscle cell fate and plasticity. Nature 460: 705-710.

Dimmeler S and Zeiher AM (2004). Vascular repair by circulating endothelial progenitor cells: the missing link in atherosclerosis? J. Mol. Med. 82: 671-677.

Jeyaseelan K, Lim KY and Armugam A (2008). MicroRNA expression in the blood and brain of rats subjected to transient focal ischemia by middle cerebral artery occlusion. Stroke 39: 959-966.

Jickling GC and Sharp FR (2011). Blood biomarkers of ischemic stroke. Neurotherapeutics 8: 349-360.

Lagos-Quintana M, Rauhut R, Lendeckel W and Tuschl T (2001). Identification of novel genes coding for small expressed RNAs. Science 294: 853-858.

Lloyd-Jones D, Adams R, Carnethon M, De Simone G, et al. (2009). Heart disease and stroke statistics-2009 update: a report from the American Heart Association Statistics Committee and Stroke Statistics Subcommittee. Circulation 119: e21-181. 
Long X, Bell RD, Gerthoffer WT, Zlokovic BV, et al. (2008). Myocardin is sufficient for a smooth muscle-like contractile phenotype. Arterioscler. Thromb. Vasc. Biol. 28: 1505-1510.

Luttun A, Tjwa M, Moons L, Wu Y, et al. (2002). Revascularization of ischemic tissues by PlGF treatment, and inhibition of tumor angiogenesis, arthritis and atherosclerosis by anti-Flt1. Nat. Med. 8: 831-840.

Owens GK (1995). Regulation of differentiation of vascular smooth muscle cells. Physiol. Rev. 75: 487-517.

Tan KS, Armugam A, Sepramaniam S, Lim KY, et al. (2009). Expression profile of MicroRNAs in young stroke patients. PLoS One 4: e7689. 